\title{
ESTIMATION OF 4th AND 6th ORDER ODF COEFFICIENTS FROM ELASTIC PROPERTIES IN COLD ROLLED STEEL SHEETS
}

\author{
KEI SAKATA, DOMINIQUE DANIEL and JOHN J. JONAS \\ Department of Mining and Metallurgical Engineering, McGill University, 3450 \\ University St., Montreal, Québec H3A 2 A7 Canada
}

(Received February 13, 1989)

\begin{abstract}
Generally only the 4th order ODF coefficients are deduced from the observed elastic anisotropy of textured polycrystalline materials with cubic/orthorhombic symmetry. In this study, a method is described for the prediction of the 4th and 6th order ODF coefficients from the elastic properties of cold rolled and annealed steel sheets of 5 different types. In order to link these properties with the texture, the elastic energy method of Bunge (1974) is employed. By estimating the volume fractions of the principal preferred orientations and their gaussian spreads, ODF coefficients of the 4 th $\left(C_{4}^{11}, C_{4}^{12}\right.$ and $\left.\mathrm{C}_{4}^{13}\right)$ and 6th $\left(\mathrm{C}_{6}^{11}, \mathrm{C}_{6}^{12}\right.$ and $\left.\mathrm{C}_{6}^{14}\right)$ orders can be successfully obtained. As a result, the planar $r$-value distribution can be predicted more accurately than when only the 4th order coefficients are employed.
\end{abstract}

KEY WORDS Elastic properties, cold rolled steel, ODF coefficients, volume fractions, on-line prediction of $r$-value, elastic energy method

\section{INTRODUCTION}

It is of considerable industrial interest to develop on-line equipment and methods for $r$-value prediction from ultrasonic measurements. This is one reason for the evolution and improvement of the electromagnetic acoustic transducer (EMAT). However, such equipment only leads to partial texture characterization, because normally only three ODF coefficients (for $l=4$ ) can be estimated from ultrasonic velocities, while the number necessary for calculation of the plastic anisotropy using the series expansion method is considerably higher. For example, in textures of commercial steels, it is necessary to go to $l=10$ or 12 for $r$-value convergence to be obtained. It is also possible to assess the elastic properties using Modul-R equipment (Mould and Johnson, 1973), but this method is not nondestructive, and so cannot be used on-line. The latter was originally developed as an $r$-value sensor, using equations to correlate $\bar{r}$-value with $\bar{E}$ and $\Delta r$ with $\Delta E$, where $E$ is Young's modulus and - and $\Delta$ represent averaging and planar anisotropy, respectively, defined by $\bar{X}=\left(X_{0}+2 X_{45}+X_{90}\right) / 4$ and $\Delta X=$ $\left(X_{0}-2 X_{45}+X_{90}\right) / 2$. The principles of this technique were clarified by Davies $e t$ al. (1972) using the series expansion method.

Statistically exact solutions for Young's modulus in polycrystalline materials were described by Kröner (1958) for the case of random orientations, and by Morris (1970) for generalized textures. These are most realistic, but too time 
consuming for on-line applications. A method for the second order approximation of Young's modulus was proposed by Bunge (1974), which is based on the minimization of elastic energy (referred to below as the elastic energy method). This technique leads to a much narrower range of estimates than that defined by the Voigt (1928) and Reuss (1929) assumptions, and displays good agreement with Kröner type calculations. Theoretically, twelve ODF coefficients up to $l=8$ are necessary to perform the Young's modulus calculation by this method.

In this study, the sensitivity of the elastic energy method to the values of the higher order coefficients $(l=6$ and 8$)$ is analyzed in some detail. The 4th order coefficients are then calculated from the Young's modulus data using the elastic energy method. With the aid of estimated volume fractions of the principal preferred orientations, some of the 6th order terms are obtained as well. It is shown that the availability of these higher order coefficients can lead to more accurate $r$-value predictions from acoustic measurements than when solely 4th order coefficients are employed.

\section{EXPERIMENTAL WORK}

Eighteen commercial low carbon steel sheets of 5 different types were studied: i) batch annealed Al-killed drawing quality (AKDQ) steel, ii) commercial grade rimmed steel, iii) high strength low alloy (HSLA) steel, and iv) and v) two types of interstitial free extra low carbon steel. In the first type of interstitial free steel (IF1), the $\bar{r}$-values fall in the range from 1.5 to 1.9 and the $\Delta r$ 's of 0.2 to 0.4 are less than those of the AKDQ steels. In the second type (IF2), the $\bar{r}$-values are above 2.0 and the $\Delta r$ 's are still smaller, ranging from -0.2 to 0.2 .

The values of Young's modulus were measured at every $15^{\circ}$ in the rolling plane

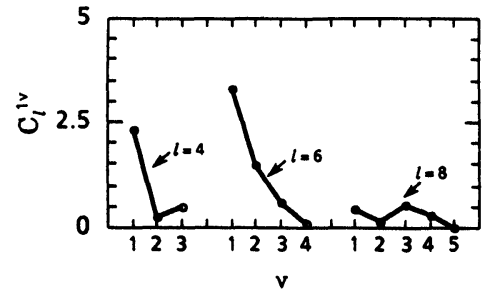

(a) AKDQ

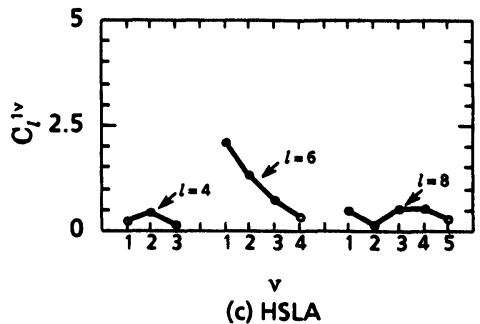

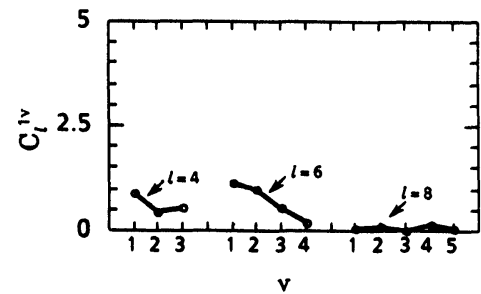

(b) rimmed

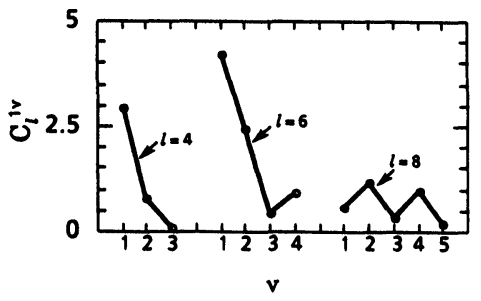

(d) IF2

Figure 1 Absolute values of the ODF coefficients up to $l=8$ determined from the X-ray measurements in the (a) AKDQ, (b) rimmed, (c) HSLA and (d) IF2 steels. 
using Modul-R equipment. This induces a resonant frequency in the sheet specimen, from which Young's modulus can be calculated directly. According to Mould and Johnson (1973), the uncertainty in the Modul-R frequency measurements is $0.07 \%$, which corresponds to $0.14 \%$ for Young's modulus.

ODF coefficients were then determined by means of X-ray measurements, using standard techniques. Three of the steels were also analyzed using neutron diffraction, which led to reasonably good agreement with the X-ray results. Some examples of absolute values of the ODF coefficients up to $l=8$ pertaining to each type of steel are given in Figure 1 for the (a) AKDQ, (b) rimmed, (c) HSLA and (d) IF2 steels. Here it can be seen that, excluding the 4th order coefficients, $C_{6}^{11}$ has the highest value, followed by $C_{6}^{12}$, and that $C_{6}^{14}$ only has a significant value in the IF2 steel.

\section{ELASTIC ENERGY METHOD}

\section{a. Fundamentals of the method}

According to this method, proposed by Bunge (1974), the stresses $\sigma_{i}(g)$, strains $\varepsilon_{j}(g)$ and stiffnesses $C_{i j}(g)$ pertaining to crystals of orientation $g$ are given as the sums of an orientation independent mean value $(-)$ and an orientation dependent fluctuation term $(\Delta)$ as follows:

$$
\begin{aligned}
\sigma_{i}(g) & =\bar{\sigma}_{i}+\Delta \sigma_{i}(g) \\
\varepsilon_{j}(g) & =\bar{\varepsilon}_{j}+\Delta \varepsilon_{j}(g) \\
C_{i j}(g) & =\bar{C}_{i j}+\Delta C_{i j}(g)
\end{aligned}
$$

The substitution of Eqs. (1) to (3) into Hooke's law leads to

$$
\bar{\sigma}_{i}=\bar{C}_{i j} \bar{\varepsilon}_{j}+\int \Delta C_{i j}(g) \Delta \varepsilon_{j}(g) f(g) d g
$$

where $f(g)$ is the orientation distribution function or ODF. Assuming that $\Delta \varepsilon_{j}(g)=B_{j k}(g) \bar{\varepsilon}_{k}$ and that the effective elastic constants $\tilde{C}_{i j}$ are defined by $\bar{\sigma}_{i}=C_{i j} \bar{\varepsilon}_{j}$, one obtains

$$
\tilde{C}_{i j}=\bar{C}_{i j}+\int \Delta C_{i k}(g) B_{k j}(g) f(g) d g
$$

The quantities $B_{k j}(g)$ and hence the strains $\Delta \varepsilon_{j}(g)$ can be adjusted so as to minimize the mean elastic energy;

$$
E=\frac{1}{2} \int C_{i j}(g) \varepsilon_{i}(g) \varepsilon_{j}(g) f(g) d g=\min .
$$

The orientation dependent functions $\Delta C_{i k}(g)$ and $B_{k j}(g)$ can be developed into a series of generalized spherical harmonics with cubic/orthorhombic symmetry. Since products of the two 4th order generalized spherical harmonics associated with $\Delta C_{i k}$ and $B_{k j}$ are present, the effective elastic constants $\tilde{C}_{i j}$ can be 
represented as functions of the ODF coefficients, $C_{l}^{\mu v}$, up to $l=8$, as follows,

with

$$
\tilde{C}_{i j}=\bar{C}_{i j}-81 \bar{C}_{t k}^{-1} C_{a}^{2} \sum_{v, v^{\prime}=0(2)}^{4} a_{4}^{1 v}(i k) a_{4}^{1 v^{\prime}}(j t) F\left(v, v^{\prime}\right)
$$

$$
\begin{array}{r}
F\left(v, v^{\prime}\right)=\sum_{l=0}^{8} \frac{1}{2 l+1} \sum_{n=v+v^{\prime} \text { or }\left|v-v^{\prime}\right|}\{4,4,1,1 \mid l ; 1\}_{c}\left\{4,4, v, v^{\prime} \mid l, n\right\}_{r h} \\
\times C_{l}^{1 n}-\frac{C_{4}^{1 v} C_{4}^{1 v^{\prime}}}{81}
\end{array}
$$

The coefficients $a_{4}^{1 v}$ in Eq. (7) and both $\{4,4,1,1 \mid l, 1\}_{c}$ for cubic symmetry and $\left\{4,4, v, v^{\prime} \mid l, n\right\}_{r h}$ for orthorhombic symmetry in Eq. (8) are all numerical constants available in the original paper. $C_{a}$ is the elastic anisotropy of a single crystal defined by $C_{a}=C_{11}^{0}-C_{12}^{0}-2 C_{44}^{0}$, where $C_{i j}^{0}$ are the single crystal stiffnesses. Similarly, by substituting the compliances $S_{i j}$ for $C_{i j}$, the effective elastic constants $\tilde{S}_{i j}$ can be estimated. $\bar{C}_{i j}$ in Eq. (7) and $\bar{S}_{i j}$ implicit in the representation of $\tilde{S}_{i j}$ correspond to the conventional Voigt and Reuss assumptions, respectively. Their calculation by conventional processes requires ODF coefficients up to $l=4$. In the elastic energy method, the stiffnesses and compliances are "corrected" by a second order term containing the function $F\left(v, v^{\prime}\right)$ in which the 4th, 6th and 8th orders appear.

The effective elastic constants $\tilde{C}_{i j}$, $\tilde{S}_{i j}$ and their mathematical averages lead to the planar distributions of Young's modulus pertaining to the respective Voigt, Reuss and Hill (1952) type solutions. It is a characteristic of the elastic energy method that 12 independent ODF coefficients ( 3 for $l=4,4$ for $l=6$ and 5 for $l=8)$ are necessary for the calculation of Young's modulus, instead of only 3 for the conventional approach.

\section{b. Application of the method to the materials studied}

The ODF coefficients up to $l=8$ determined from the X-ray measurements were first used to calculate the dependence of Young's modulus on orientation in the sheet. These calculations were carried out in six different ways. The first three involved the use of the Voigt, Reuss and Hill assumptions in the classical way, using only the 4th order coefficients. The last three were carried out by the elastic energy method and thus employed twelve coefficients pertaining to the 4th, 6th and 8th orders. Examples of the results obtained are given in Figure 2 for the (a) AKDQ, (b) rimmed, (c) HSLA and (d) IF2 steels. The single crystal elastic constants employed here are $C_{11}^{0}=230 \mathrm{GPa}, C_{12}^{0}=136 \mathrm{GPa}$ and $C_{44}^{0}=116.5 \mathrm{GPa}$; these were selected from a review of literature data for iron and mild steel (Ledbetter and Reed, 1973).

It is of interest that the Voigt and Reuss elastic energy calculations define a considerably narrower range than the classical one and that the Hill elastic energy estimate leads to values which are very close to the experimental ones. Although uncertainties in the single crystal elastic constants can affect the magnitude of Young's modulus (Daniel et al., 1988), it can nevertheless be concluded that the Hill approximation combined with the elastic energy method leads to more accurate predictions than the classical one. This is because, according to Bunge 


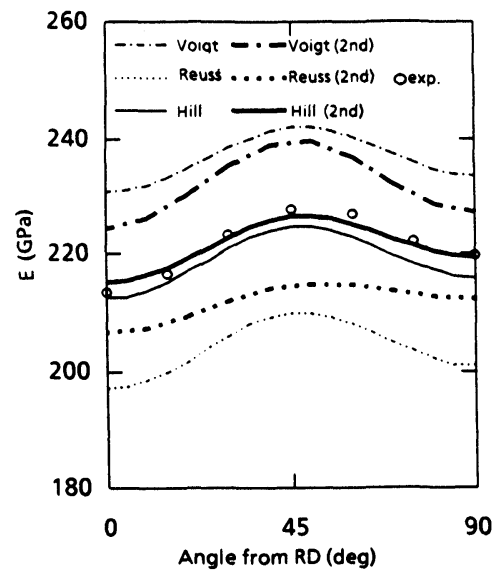

(a) AKDQ

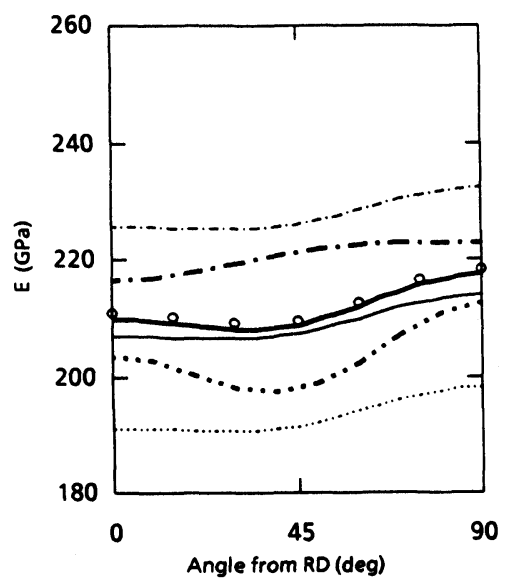

(c) HSLA

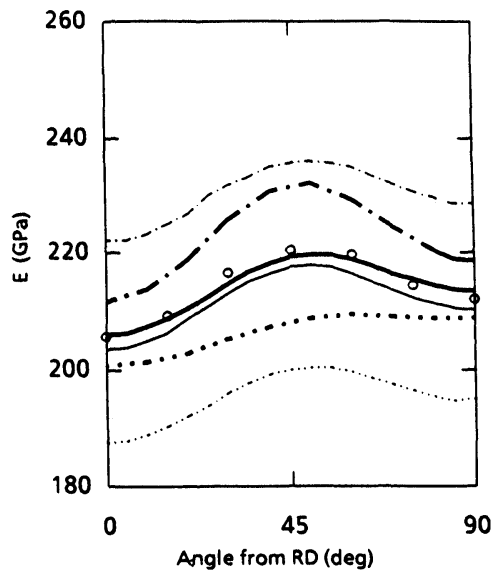

(b) rimmed

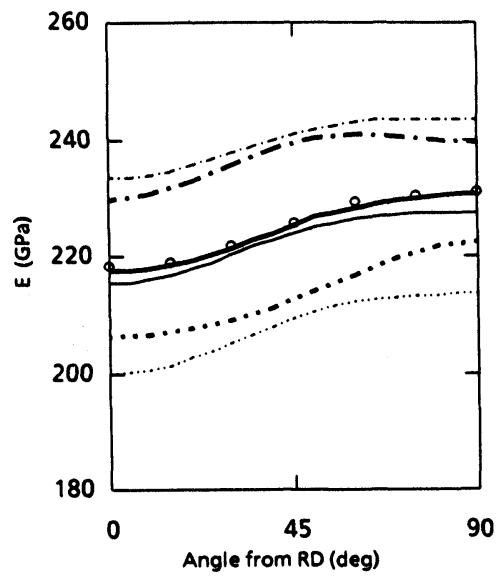

(d) IF2

Figure 2 Planar distributions of Young's modulus derived from the classical Voigt, Reuss and Hill approximations and those based on the elastic energy method in the (a) AKDQ, (b) rimmed, (c) HSLA and (d) IF2 steels.

(1974), this technique leads to close coincidence with the theoretically exact Kröner type solution for random orientations.

\section{ESTIMATION OF 4TH AND 6TH ORDER ODF COEFFICIENTS}

\section{a. Contribution of higher order ODF coefficients to those of the 4th order}

It is evident from Eqs. (7) and (8) that the 4th order coefficients play the most important role in determining the values of the polycrystal stiffnesses and compliances. However, the higher order coefficients can also affect the Young's 
Table 1 Standard deviations of the 4th order ODF coefficients calculated by the elastic energy method in 18 steels

\begin{tabular}{llll}
\hline Case & $C_{4}^{11}$ & $C_{4}^{12}$ & $C_{4}^{13}$ \\
\hline A & 0.40 & 0.11 & 0.25 \\
B & 0.39 & 0.09 & 0.13 \\
C & 0.40 & 0.11 & 0.14 \\
\hline
\end{tabular}

A: 6th and 8th order ODF coefficients are disregarded.

B: 6th and 8th order ODF coefficients determined from the X-ray measurements are used.

C: Only $C_{6}^{11}$ determined from the X-ray measurements is used.

modulus distribution if their values are sufficiently high. If their effects can be characterized, the inverse operation can become possible; that is to say, ODF coefficients of the 6th or even 8th order might be calculated from elastic data. For this reason, an assessment was first made of the relative importance of the four 6th order coefficients in the present materials using the X-ray data. In the first calculation, case $A$ in Table 1 , the 4th order ODF coefficients were derived from the Young's modulus data for all 18 steels on the assumptions that the 6th and 8th order coefficients are all zero. The standard deviations between the coefficients derived on the basis of the elastic energy method and those determined from the X-ray measurements are listed for each coefficient in the table.

The results of this calculation can be compared with those of case $\mathrm{B}$, in which by contrast $X$-ray based values of the 6th and 8th order coefficients were employed in the calculation. It can be seen that the error on $C_{4}^{13}$ is now halved, although those on $C_{4}^{11}$ and $C_{4}^{12}$ are only slightly reduced. This indicates that the inclusion of the higher order coefficients can improve the accuracy of prediction of the 4th order coefficients. (The large error on $C_{4}^{11}$ may be due to the uncertainties associated with the determination of both the single crystal elastic constants as well as the X-ray based ODF coefficients.) Finally, in case $C$, the calculations were repeated, but with only the $C_{6}^{11}$ coefficient being taken into account. This was justified in that it can be seen from Figure 1 that the largest higher order coefficient is $C_{6}^{11}$, the second largest is $C_{6}^{12}$, and that the others are all relatively small. From Table 1 , it is evident that most of the improvement in accuracy gained by including the higher order coefficients can be obtained from the use of the $C_{6}^{11}$ term alone.

\section{b. Introduction of volume fraction and gaussian spread}

The distribution of Young's modulus in the rolling plane is given by the following equation:

$$
E(\alpha)^{-1}=S_{11} \cos ^{4} \alpha+S_{22} \sin ^{4} \alpha+\left(S_{44}+\frac{1}{2} S_{12}\right) \sin ^{2}(2 \alpha)
$$

This expression involves three independent variables corresponding to isotropic, two-fold and four-fold symmetry terms. It is therefore mathematically impossible to calculate four (or more) ODF coefficients from experimental Young's modulus 
data using the elastic energy method without some additional information. The present approach involves first estimating the three 4th order coefficients from the experimental measurements. The values of the 4th order coefficients are then used to decompose the texture into its main ideal orientations. The volume fractions of the preferred orientations are used in turn to estimate the 4th and 6th order coefficients.

The relation between the $C_{4}^{11}$ and $C_{6}^{11}$ coefficients derived from the X-ray measurements is presented in Figure 3 . Here it can be seen that, except for the HSLA steels, all the results fall on a single line. Moreover, the equation of this line is $C_{6}^{11}=(-5.94) /(-3.94) \times C_{4}^{11}$, which figures represent the $C_{6}^{11}$ and $C_{4}^{11}$ coefficients of the $\{111\}\langle u v w\rangle$ texture (see Table 2 below). The higher the fraction of $\{111\}\langle u v w\rangle$ present, the higher the absolute value of $C_{4}^{11}$. In the case of the HSLA steels, the results are affected by the presence of a $\{100\}\langle u v w\rangle$ component, as indicated by the ODF, which gives values of about 4 and 8 , respectively, for $\{111\}\langle 011\rangle$ and $\{100\}\langle 011\rangle$. It can be shown that the addition of a $\{100\}\langle u v w\rangle$ orientation to $\{111\}\langle u v w\rangle$ displaces a given point in Figure 3 vertically downwards.

These observations suggest that $C_{6}^{11}$ can be successfully estimated from knowledge of the volume fractions of the main orientations, particularly those of the $\{111\}$ and $\{100\}$ components. For this purpose, it was decided to consider the following components: $\{111\}\langle u v w\rangle,\{100\}\langle u v w\rangle$ and $\{110\}\langle u v w\rangle$, which is a third important type of orientation in cold rolled and annealed steel sheets. These are widely separated in the inverse pole figure. From knowledge of the three 4th order coefficients, the texture can be described quantitatively in terms of these three ideal texture components. This leads to the resolution of the following equation:

$$
C_{l}^{\mu v}=\sum_{i=1}^{n} V_{i}(g) C_{l}^{\mu v}(g)
$$

where $n, V_{i}(g)$ and $C_{l}^{\mu \nu}(g)$ are, respectively, the number of orientations $g$, their individual volume fractions and their ODF coefficients.

The 4th and 6th order ODF coefficients pertaining to the principal preferred orientations and for a gaussian spread of $10^{\circ}$ are listed in Table 2. The $\{211\}\langle 011\rangle$ and $\{554\}\langle 225\rangle$ components are included here as they will be employed below for calculating $C_{6}^{12}$ and $C_{6}^{14}$. Since the 4th order coefficients do

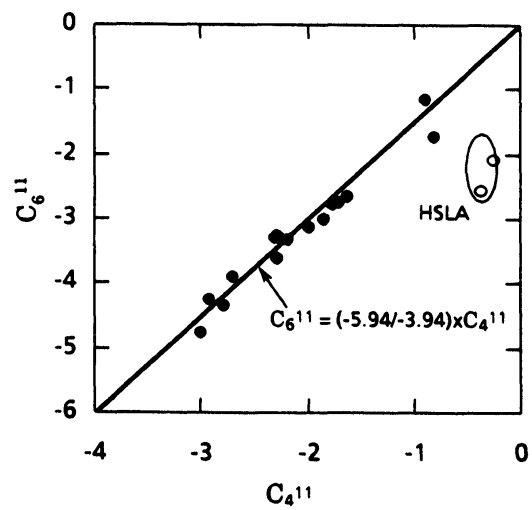

Figure 3 Relation between the values of $C_{4}^{11}$ and $C_{6}^{11}$ determined from the $\mathrm{X}$-ray measurements. Figures in the equation refer to the $C_{6}^{11}$ and $C_{4}^{11}$ coefficients pertaining to the $\{111\}\langle u v w\rangle$ component (see Table 2). 
Table 2 4th and 6th order ODF coefficients of the principal ideal orientations for a gaussian spread of $10^{\circ}$.

\begin{tabular}{lllllllll}
\hline & $\{100\}\langle 001\rangle$ & $\{100\}\langle 011\rangle$ & $\{110\}\langle 001\rangle$ & $\{110\}\langle 110\rangle$ & $\{211\}\langle 011\rangle$ & $\{554\}\langle 225\rangle$ & $\{111\}\langle 110\rangle$ & $\{111\}\langle 112\rangle$ \\
\hline$C_{4}^{11}$ & 5.90 & 5.90 & -1.48 & -1.48 & -1.48 & -3.75 & -3.94 & -3.94 \\
$C_{4}^{12}$ & 0 & 0 & -6.60 & 6.60 & -2.20 & -1.36 & 0 & 0 \\
$C_{4}^{13}$ & 4.99 & -4.99 & 3.74 & 3.74 & -2.91 & 0.94 & 0 & 0 \\
$C_{6}^{11}$ & -3.34 & -3.34 & 5.43 & 5.43 & -1.72 & -5.28 & -5.94 & -5.94 \\
$C_{6}^{12}$ & 0 & 0 & -3.02 & 3.02 & 6.39 & 1.04 & 0 & 0 \\
$C_{6}^{13}$ & 8.84 & -8.84 & -5.52 & -5.52 & 1.60 & -1.46 & 0 & 0 \\
$C_{6}^{14}$ & 0 & 0 & -4.49 & 4.49 & 4.15 & -5.25 & 5.32 & -5.32 \\
\hline
\end{tabular}

not allow the particular ideal orientations forming the $\{111\}$ fiber to be distinguished, the $\{111\}\langle u v w\rangle$ component, without specification of $\langle u v w\rangle$, was one of the unknowns. For the $\{100\}\langle u v w\rangle$ and $\{110\}\langle u v w\rangle$ components, two specific directions, $\langle 001\rangle$ and $\langle 011\rangle$, were selected. In this way, Eq. (10) was solved for the four possible hypothetical cases based on the five components; that is, the ones obtained by omitting in turn those containing the $\langle 001\rangle$ and $\langle 011\rangle$ directions from the $\{100\}$ and $\{110\}$ components. In every case studied, only one of the four descriptions gave a permissible solution for $V_{i}$ corresponding to volume fractions between $0 \%$ and $100 \%$. From this solution, the amount of the random component was defined as $100 \%-\left(V_{\{111\}}+V_{\{100\}}+V_{\{110\}}\right)$.

In order to give a realistic description of the texture, a gaussian spread $\omega$ was employed about each ideal orientation. The associated ODF coefficients were obtained from the following relation (Bunge and Esling, 1982),

$$
C_{l}^{\mu v}=\frac{\exp \left(-l^{2} \omega^{2} / 4\right)-\exp \left\{-(l+1)^{2} \omega^{2} / 4\right\}}{1-\exp \left(-\omega^{2} / 4\right)} \dot{T}_{l}^{\mu v}
$$

where $\dot{T}_{l}^{\mu v}$ are the cubic/orthorhombic generalized spherical harmonics. To carry out the calculation, a value has to be selected for $\omega$, which normally lies between 5 and $20^{\circ}$. The three 4th order coefficients temporarily calculated from the Young's modulus data (neglecting the 6th and 8th order coefficients, as in case A in Table 1), can be represented in Eq. (10) as functions of the volume fractions of the three orientations and the gaussian spread. These are displayed in Figure 4

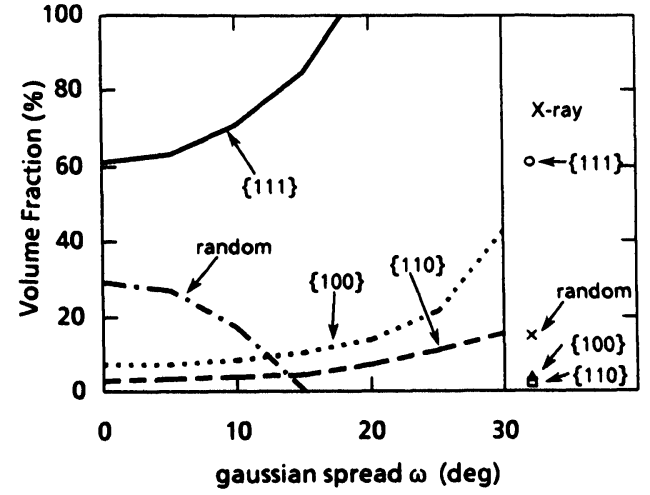

Figure 4 Effect of gaussian spread $\omega$ on the volume fractions of the $\{111\}\langle u v w\rangle$, $\{110\}\langle 001\rangle$ or $\langle 110\rangle,\{100\}\langle 001\}$ or $\langle 011\rangle$ and random components in an AKDQ steel calculated according to the present method. The volume fractions determined from the $\mathrm{X}$-ray measurements are shown along the right margin. 
for an AKDQ steel. The volume fractions determined from the ODF are shown along the right hand edge of the figure for purposes of comparison. It is evident that a gaussian spread of about $10^{\circ}$ leads to good agreement between the volume fractions calculated from the elastic data and those derived from the ODF. Similar results were obtained for the other types of steel. For this reason, a spread of $10^{\circ}$ was adopted for all the calculations described below.

\section{c. Estimating the 4th order ODF coefficients and $C_{6}^{11}$ by the elastic energy method}

On the basis of a $10^{\circ}$ spread around the ideal components, the experimental elastic data and the initial set of 4th order coefficients evaluated from the elastic energy method, a first estimate was made of $C_{6}^{11}$. The 4th order coefficients were then recalculated by means of the elastic energy method using the derived value of $C_{6}^{11}$ (case $C$ of Table 1 ). This cycle was repeated until a convergent solution was obtained, generally in the second iteration.

The $C_{4}^{11}, C_{4}^{12}, C_{4}^{13}$ and $C_{6}^{11}$ coefficients obtained in this way are compared with those determined from the X-ray measurements in Figure 5. The error bars shown in the figures are associated with the uncertainties involved in the use of the Modul-R and X-ray equipment. With regard to the 4th order coefficients,

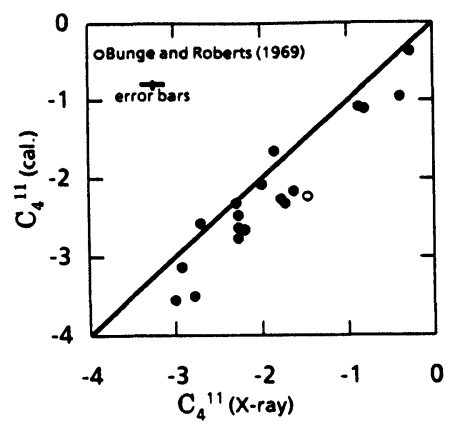

(a)

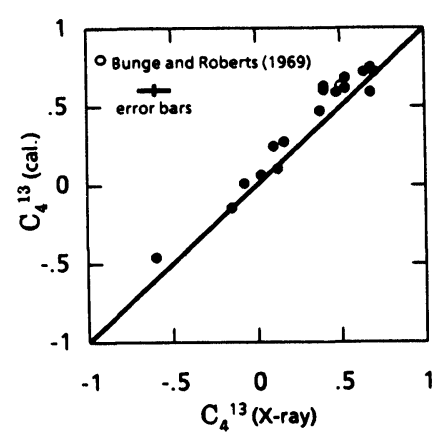

(c)

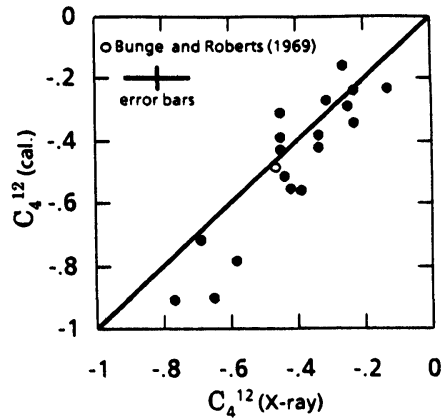

(b)

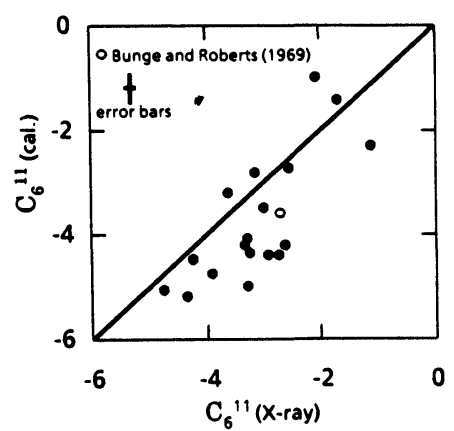

(d)

Figure 5 Comparison of the ODF coefficients determined in this study and those measured by X-ray methods: (a) $C_{4}^{11}$, (b) $C_{4}^{12}$, (c) $C_{4}^{13}$ and (d) $C_{6}^{11}$. The symbol $(O)$ refers to values derived from literature data on an AKDQ steel (Bunge and Roberts, 1969); here their Young's modulus data were converted into ODF coefficients as described in the text. 
only the $C_{4}^{11}$ fit is shifted beyond the error ranges, although it remains approximately parallel to the $x=y$ line. This seems to be related to the inaccuracies associated with the values of the single crystal elastic constants that were used (Daniel et al., 1988). The standard deviations are almost equal to those associated with condition B in Table 1, i.e. the most ideal case. Some scatter is observed in Figure 5(d), so the correlation coefficient pertaining to $C_{6}^{11}$ is 0.75 . This is mainly because of the presence of the minor orientations, which are ignored in this calculation, together with a contribution from the inaccuracy of the single crystal elastic constants. Literature data for Young's modulus in an AKDQ steel (Bunge and Roberts, 1969) were also used to calculate the ODF coefficients in a similar manner. Their results are represented by the symbol $(O)$ in the figures, and are in good agreement with those obtained from the materials used in this study.

A similar method of estimating the volume fractions of the three main components from acoustoelastically obtained 4th order ODF coefficients has been described by Hirao et al. (1988). However, their method does not lead to close estimates of $C_{6}^{11}$, probably because they did not take the gaussian spread into account.

\section{d. Estimating $C_{6}^{12}$ by the elastic energy method}

Other preferred orientations found in cold rolled and annealed steel sheets are the $\{211\}\langle 011\rangle$ and the $\{554\}\langle 225\rangle$, the latter often appearing in IF steels. These can contribute significantly to the values of the other 6th order coefficients (in addition to $\left.C_{6}^{11}\right)$. However, the unique solutions for the volume fractions of five orientations $(\{111\}\langle u v w\rangle,\{100\}\langle 001\rangle$ or $\langle 011\rangle,\{110\}\langle 001\rangle$ or $\langle 110\rangle$, $\{211\}\langle 011\rangle$ and $\{554\}\langle 225\rangle)$ cannot be obtained through relations such as Eq. (10) using four independent variables (the $C_{4}^{11}, C_{4}^{12}, C_{4}^{13}$ and $C_{6}^{11}$ coefficients), unless a suitable additional assumption is made.

The relation between the fraction of the random component and $C_{4}^{11}$, as determined from X-ray data, is shown in Figure 6 . It should be noted that as the absolute value of $C_{4}^{11}$ increases, i.e. as the $\{111\}$ component increases in intensity, the random intensity decreases. Thus the random intensity can be estimated from the value of $C_{4}^{11}$. In the calculation described below, the random fraction was

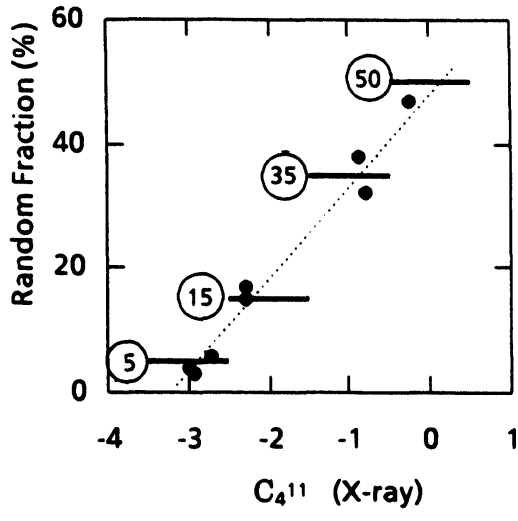

Figure 6 Relation between volume fraction of the random component and $C_{4}^{11}$, both determined from the $\mathrm{X}$-ray measurements. The numbers in the circles were adopted as the scale for random intensity for the present calculations. 
assumed to have the value derived from this correlation. Then four versions of Eq. (10) were solved corresponding to the decomposition of the four coefficients in a manner analogous to that employed above in section 4.b. The fifth equation which allows the resolution for the volume fractions $V_{i}$ is:

$$
V_{\{111\}\langle u v w\rangle}+V_{\{100\}\langle u v w\rangle}+V_{\{110\}\langle u v w\rangle}+V_{\{554\}\langle 225\rangle}+V_{\{112\}\langle 110\rangle}=100-V_{\text {random }}
$$

With the aid of Eq. (10), the values of $C_{6}^{12}$ shown in Figure 7(a) were calculated in this way. Although there is considerable scatter, a clear trend can nevertheless be seen. A further result calculated from the Young's modulus data of Bunge and Roberts (1969) is included in the figure, and is in good agreement with that derived from the X-ray measurements. With respect to $C_{6}^{13}$, the values determined from the X-ray data fell in a narrow range of values from -0.4 to -0.8 . Thus, no particular trend could be established for the present collection of steels.

\section{e. Estimation of the $C_{6}^{14}$ coefficient}

We proceed finally to the calculation of $C_{6}^{14}$. Here the $C_{6}^{12}$ results were employed and it was further assumed, as discussed later, that the volume fraction ratio of the $\{111\}\langle 110\rangle$ to the $\{111\}\langle 112\rangle$ component is two. Needless to say, this change does not affect the previous results. With the aid of the volume fractions of the five components, the values of $C_{6}^{14}$, displayed in Figure 7(b), were calculated using Eq. (9). It can be seen that the agreement is relatively good for most of the steels. Only the IF2 steels have significant (non-zero) values of the X-ray based $C_{6}^{14}$, as shown in Fig. 1. This trend is confirmed by the present calculation and can be used for predictive purposes. The result derived from the literature (Bunge and Roberts, 1969) falls in the same range as the present AKDQ steels. By contrast, in the case of the IF2 steels, the calculated $C_{6}^{14}$ values are considerably lower than those derived from the X-ray data. This discrepancy seems to be again linked to the use of values of the single crystal constants which are inappropriate to these steels.

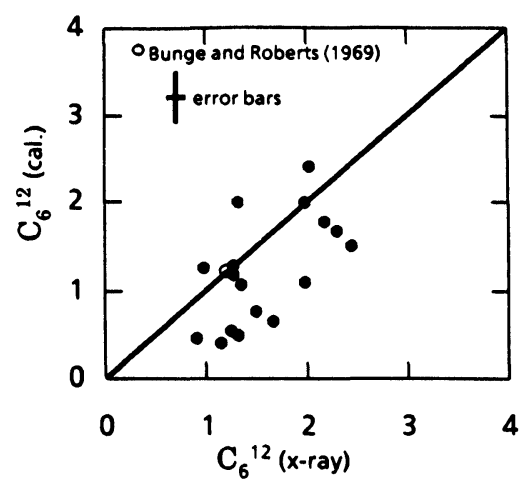

(a)

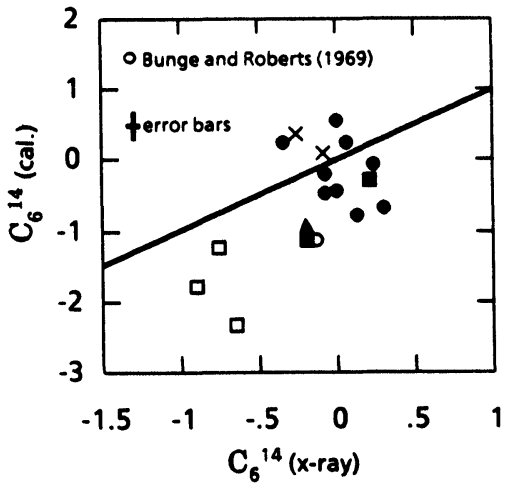

(b)

Figure 7 Comparison of the 6th order coefficients derived in this study and those measured by X-rays: (a) $C_{6}^{12}$ and (b) $C_{6}^{14}$. The symbol $(O)$ refers to data on an AKDQ steel derived from the literature (Bunge and Roberts, 1969). Legend for Figure 7b: (O)-AKDQ; (A)-rimmed; ( $\times$ )-HSLA; (ם)-IF1; and ( $\square$ )-IF2 steels. 


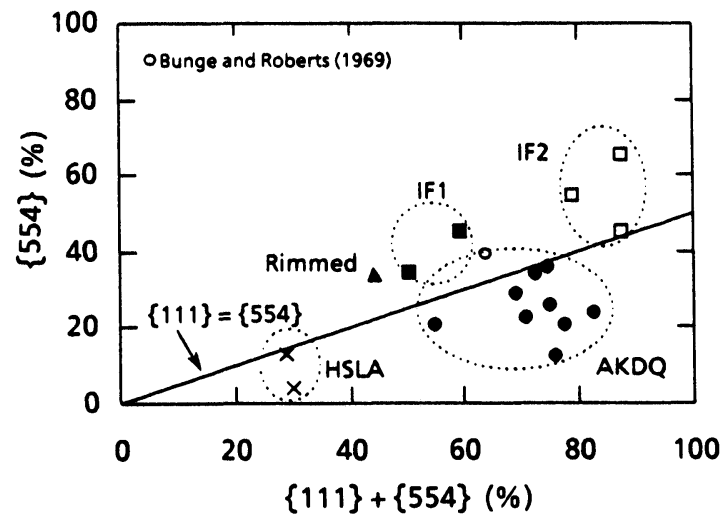

Figure 8 Comparison of the volume fractions of $\{554\}\langle 225\rangle$ and of the sum of $\{111\}\langle u v w\rangle$ and $\{554\}\langle 225\rangle$ obtained from the $C_{6}^{14}$ calculations.

According to Table 2, the $C_{6}^{14}$ coefficient is strongly affected by the presence of the $\{554\}\langle 225\rangle$ component. The calculated volume fractions of the $\{111\}\langle u v w\rangle$ and $\{554\}\langle 225\rangle$ components are displayed in Figure 8 . The sheets with high $\bar{r}$-values, such as the AKDQ and IF steels, reveal high volume fractions of the sum of the $\{111\}\langle u v w\rangle$ and $\{554\}\langle 225\rangle$ components, as expected. Furthermore, the IF2 steels, which have higher $\bar{r}$-values and very low $\Delta r$ 's, have the highest volume fractions of $\{554\}\langle 225\rangle$.

These tendencies are qualitatively consistent with those deduced from the X-ray based ODF's. The intensities at $\Psi_{2}=45^{\circ}$ and $\Phi=55^{\circ}$, which correspond to

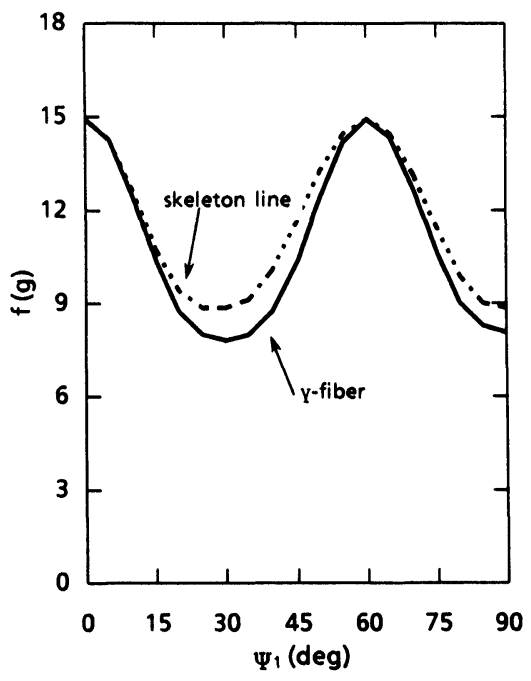

(a) AKDQ

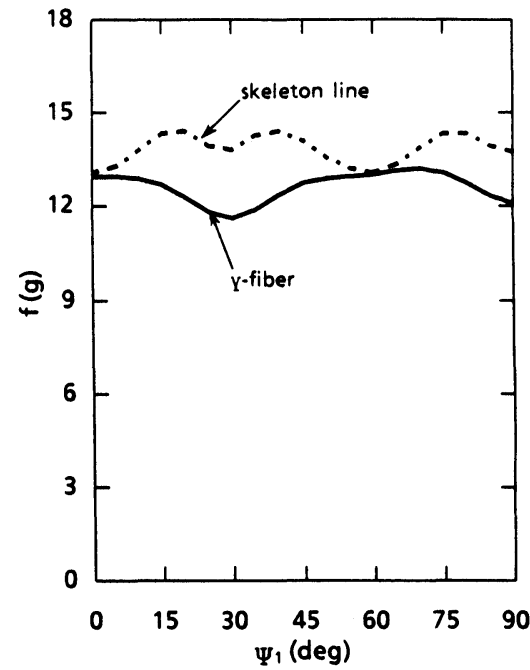

(b) IF2

Figure 9 Comparison of $f(g)$ along the $\gamma$-fiber and the skeleton line in the (a) AKDQ and (b) IF2 steels obtained from the X-ray data. These are displayed in the $\Psi_{2}=45^{\circ}$ section. 


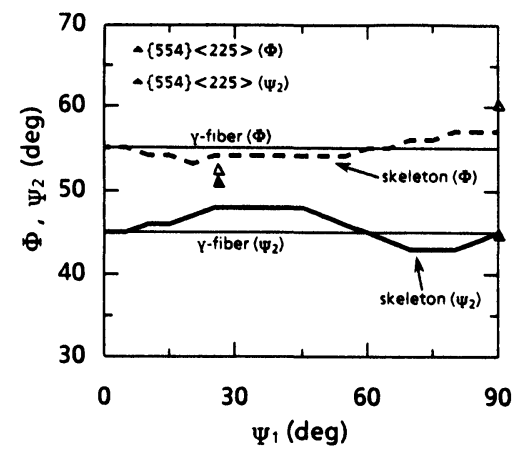

(a) AKDQ

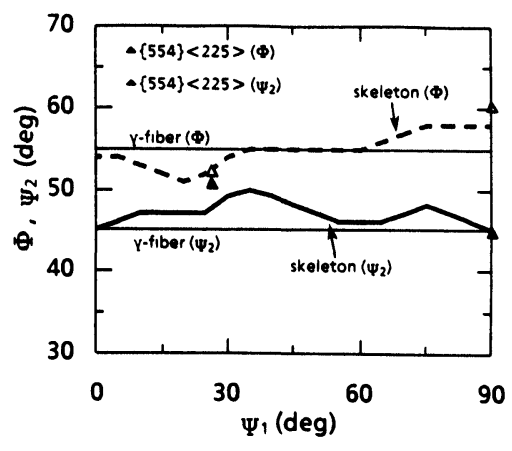

(b) IF2

Figure 10 Locations of the skeleton lines in the (a) AKDQ and (b) IF2 steels of Figure 9.

the $\gamma$-fiber, are shown in Figure 9 for the (a) AKDQ and (b) IF2 steels. These fiber intensities are compared with those along the so-called skeleton line in the two figures. The locations of the skeleton lines along the $\Psi_{1}$ axis are shown in Figure 10. In the case of the AKDQ steel, the main orientation is $\{111\}\langle 011\rangle$ $\left(\Psi_{1}=0\right.$ and $60^{\circ}$, Figure $10 \mathrm{a}$ ), so that $f(g)$ of the $\gamma$-fiber and that of the skeleton line are almost identical. By contrast, in the case of the IF2 steel (Figure 10b), $f(g)$ along the $\gamma$-fiber does not show any significant peaks, whereas $f(g)$ along the skeleton line is higher than that along the $\gamma$-fiber, especially near the $\{554\}\langle 225\rangle$ component $\left(\Psi_{1}=26\right.$ and $\left.90^{\circ}\right)$.

As discussed above, a specific component of the $\{111\}$ fiber cannot be differentiated using the 4th order ODF coefficients together with $C_{6}^{11}$ and $C_{6}^{12}$ (see Table 2). The $C_{6}^{14}$, on the other hand, varies along the fiber, with a maximum at $\{111\}\langle 110\rangle$ and a minimum at $\{111\}\langle 112\rangle$. As shown in Figure $9, f(g)$ values near $\{111\}\langle 110\rangle$ are about twice those in the vicinity of $\{111\}\langle 112\rangle$. Even for the IF2 steels, a similar trend can be expected, if overlapping due to the gaussian spread between $\{111\}\langle 112\rangle$ and $\{554\}\langle 225\rangle$ is taken into account. This is why a two-to-one volume fraction ratio for $\{111\}\langle 110\rangle /\{111\}\langle 112\rangle$ was employed for the calculation of the $C_{6}^{14}$ coefficients.

\section{APPLICATION TO $r$-VALUE PREDICTION USING ULTRASONIC MEASUREMENTS}

This study was expressed in terms of Young's modulus, as it can be conveniently evaluated, although in a destructive way. By contrast, the ultrasonic velocities can be measured nondestructively, and are therefore more suitable for on-line purposes. The theory of acoustoelasticity in unstressed anisotropic orthorhombic polycrystalline materials has been reviewed by Sayers (1982) and Thompson et al. (1986). Linear expressions linking the 4th order ODF coefficients and the longitudinal or shear wave velocities were also developed by Sayers (1982), Lee $e t$ al. (1987) and Thompson et al. (1987). These formulae are based on the constant strain (Voigt) assumption. Similar expressions based on the Hill assumption were 


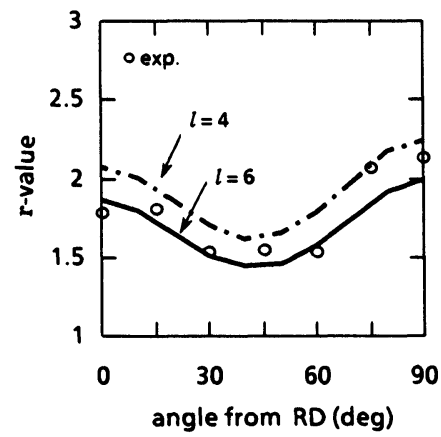

(a) AKDQ

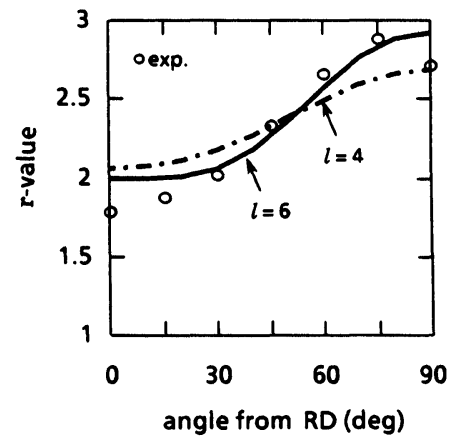

(b) IF2

Figure 11 Planar $r$-value distributions calculated from the relaxed constraint model using: i) the ODF coefficients of the 4th order (classical Hill assumption) and ii) the 4th and 6th order coefficients determined in this study. These are compared with those measured experimentally by tensile testing in the (a) AKDQ and (b) IF2 steels.

derived by Hirao et al. (1987). The comparison of the 4th order ODF coefficients derived from ultrasonic velocities with those from Young's modulus measurements in the same steels has also led to good agreement (Daniel et al., 1988). Thus, the method described here can be employed in the design of on-line equipment for $r$-value prediction. Calculations of the present type take less than 2 minutes on an IBM/PC AT.

Once the 6th order ODF coefficients are predicted accurately, in addition to those of the 4th order, more realistic calculations of plastic properties such as the $r$-value and yield surface can be made. With the aid of the 4th and 6th order ODF coefficients determined in this study, the planar $r$-value distributions in the AKDQ and IF2 steels were calculated using a relaxed constraint model (Daniel, 1989). Here, the $C_{6}^{14}$ coefficient employed was determined using a linear regression between the calculated and X-ray based $C_{6}^{14}$ values. These predictions are compared in Figure 11 with those deduced from the 4th order coefficients calculated from the classical Hill assumption and with the measurements made by tensile testing. It is evident that the 4th order coefficients alone, which have been widely employed for on-line $r$-value prediction from elastic information, lead to deviations in $r$-value from the measured ones. Use of both the 4th and 6th order coefficients, on the other hand, leads to considerably better $r$-value agreement. This improvement in the fit probably occurs because the values of the ODF coefficients over $l=8$ as well as those of the higher order Taylor factor coefficients of the series expansion are negligibly small. Another merit that arises from the possibility of determining the 6th order coefficients, especially $C_{6}^{14}$, from ultrasonic measurements is that these can be used to predict six-fold earing behavior during deep drawing.

\section{CONCLUSIONS}

A method of predicting the 6th order ODF coefficients from the elastic properties of cold rolled steel sheets has been described. It was tested on 5 different types of 
steel and employed the elastic energy method together with estimates of the volume fractions of the principal ideal orientations based on a gaussian type decomposition of the texture.

In order to represent the planar distribution of Young's modulus with the elastic energy method, twelve ODF coefficients up to $l=8$ are theoretically required. The effect of the higher order coefficients on those of the 4th order derived from Young's modulus measurements was first evaluated. It is evident that $C_{6}^{11}$ plays the most important role for 4 th order prediction. By assuming volume fractions of the three main orientations together with a gaussian spread of $10^{\circ}$, a first estimate can be made of $C_{6}^{11}$. The incorporation of this value into the calculations leads to more accurate 4th order ODF coefficients. This process can be repeated until a stable solution is obtained. In this way, $C_{6}^{11}$ can be deduced from elastic measurements, in addition to those of the 4th order. Once the four independent variables $C_{4}^{11}, C_{4}^{12}, C_{4}^{13}$ and $C_{6}^{11}$ are known, the other 6 th order coefficients can be estimated. This requires some knowledge of the approximate volume fractions of the $\{554\}\langle 225\rangle,\{211\}\langle 011\rangle$ and random components. The latter can be deduced from the value of $C_{4}^{11}$, leading to estimates of $C_{6}^{12}$ and $C_{6}^{14}$.

This method can be used to improve the accuracy of on-line equipment for $r$-value prediction employing acoustoelastic measurements. It can also detect the presence of six earing behavior, which is sometimes observed in interstitial free steels. Such a result cannot be obtained from conventional elastic methods, which only lead to the 4th order coefficients.

\section{Acknowledgements}

The authors are indebted to Dr. J. F. Bussière of NRCC-IMRI, Boucherville for his encouragement and support, to Dr. P. R. Morris for his helpful comments, and to M. McLean (Algoma Steel Corp. Ltd.), J. Thompson (Stelco Inc.) and A. Vigeant (Dofasco Inc.) for supplying the steel sheets. They express their thanks to Atomic Energy of Canada Ltd. for the X-ray and neutron diffraction measurements, and to the Canadian Steel Industry Research Association, the Natural Sciences and Engineering Research Council of Canada and the FCAR program of the Ministry of Education of Quebec for financial support. Finally, K. Sakata expresses his gratitude to the Kawasaki Steel Corp. for granting a period of study leave at McGill University as well as for texture measurements and supplying steel sheets.

\section{References}

Bunge, H. J. and Roberts, W. (1969). Orientation distribution, elastic and plastic anisotropy in stabilized steel sheet, J. Appl. Cryst., 2, 116-128.

Bunge, H. J. (1974). The effective elastic constants of textured polycrystals in second order approximation, Kristall Tech., 9, 413-423.

Bunge, H. J. and Esling, C. (1982). Quantitative Texture Analysis. Deutsche Gesellschaft für Metallkunde, 330-334.

Daniel, D., Sakata, K., Jonas, J. J., Makarow, I. and Bussière, J. F. (1988). Acoustoelastic determination of the fourth order ODF coefficients and application to $r$-value prediction, Symp. on Nondestructive Monitoring of Material Properties, MRS fall meeting, Nov. 28-Dec. 3, Boston.

Daniel, D. (1989). Ph.D. thesis, McGill University.

Davies, G. J., Goodwill, D. J. and Kallend, J. S. (1972). Elastic and plastic anisotropy in sheets of cubic metals, Metall. Trans., 3,1627-1631.

Hill, R. (1952). The elastic behavior of a crystalline aggregate, Proc. Phys. Soc., A65, 349-354. 
Hirao, M., Aoki, K. and Fukuoka, H. (1987). Texture of polycrystalline metals characterized by ultrasonic velocity measurements, J. Acoust. Soc. Am., 81, 1434-1440.

Hirao, M., Hara, N., Fukuoka, H. and Fujisawa, K. (1988). Ultrasonic monitoring of texture in cold-rolled steel sheets, J. Acoust. Soc. Am., 84, 667-672.

Kröner, E. (1958). Berechnung der elastischen Konstanten des Vielkristalles aus den Konstanten des Einkristalls, Z. Phys., 151, 504-518.

Lee, S. S., Smith, J. F. and Thompson, R. B. (1987). Inference of crystallite orientation distribution function from the velocity of ultrasonic plate modes, Proc. of Nondestructive Characterization of Materials II, edited by Bussière, J. F. et al. 555-562, Plenum Press, New York.

Ledbetter, H. N. and Reed, P. R. (1973). Elastic properties of metals and alloys, I. iron, nickel and iron-nickel alloys, J. Phys. Chem. Ref. Data, 2, 531-560.

Morris, P. R. (1970). Elastic constants of polycrystals, Int. Eng. Sci., 8, 49-61.

Mould, P. R. and Johnson, Jr. T. E. J. (1973). Rapid assessment of drawability of cold-rolled low carbon steel sheets, Sheet Met. Ind., 50, 328-333.

Reuss, A. (1929). Berechnung der Fließgrenze von Mischkristallen auf Grund der Plastizitätsbedingung für Einkristalle, Z. Angew. Math. Mech., 9, 49-58.

Sayers, C. M. (1982). Ultrasonic velocities in polycrystalline aggregates, J. Phys. D15, 2157-2167.

Thompson, R. B., Lee, S. S. and Smith, J. F. (1986). Relative anisotropies of plane wave and guided modes in thin orthorhombic plates: implication for texture characterization, J. Acoust. Soc. Am., 80, 921-931.

Thompson, R. B., Lee, S. S. and Smith, J. F. (1987). Angular dependence of ultrasonic wave propagation in a stressed, orthorhombic continuum: theory and application to the measurements of stress and texture, Ultrasonics, 25, 133-137.

Voigt, W. (1928). Lehrbuch der Kristallphysik, B. G. Teubner, Leipzig. 